\title{
Dietary supplementation of young broiler chickens with Capsicum and turmeric oleoresins increases resistance to necrotic enteritis
}

\author{
Sung Hyen Lee ${ }^{1}$, Hyun S. Lillehoj ${ }^{1 *}$, Seung I. Jang ${ }^{1}$, Erik P. Lillehoj ${ }^{2}$, Wongi Min ${ }^{3}$ and David M. Bravo ${ }^{4}$ \\ ${ }^{1}$ Animal Parasitic Diseases Laboratory, Animal and Natural Resources Institute, Agricultural Research Service-US \\ Department of Agriculture, Building 1043, BARC-East, Beltsville, MD 20705, USA \\ ${ }^{2}$ Department of Pediatrics, School of Medicine, University of Maryland, Baltimore, MD, USA \\ ${ }^{3}$ College of Veterinary Medicine, Research Institute of Life Science, Gyeongsang National University, Jinju 660-701, Republic of Korea \\ ${ }^{4}$ Pancosma S.A., Geneva, Switzerland
}

(Submitted 6 July 2012 - Final revision received 27 November 2012 - Accepted 27 November 2012 - First published online 9 April 2013)

\begin{abstract}
The Clostridium-related poultry disease, necrotic enteritis (NE), causes substantial economic losses on a global scale. In the present study, a mixture of two plant-derived phytonutrients, Capsicum oleoresin and turmeric oleoresin (XT), was evaluated for its effects on local and systemic immune responses using a co-infection model of experimental NE in commercial broilers. Chickens were fed from hatch with a diet supplemented with XT, or with a non-supplemented control diet, and either uninfected or orally challenged with virulent Eimeria maxima oocysts at $14 \mathrm{~d}$ and Clostridium perfringens at $18 \mathrm{~d}$ of age. Parameters of protective immunity were as follows: (1) body weight; (2) gut lesions; (3) serum levels of $C$. perfringens $\alpha$-toxin and NE B-like (NetB) toxin; (4) serum levels of antibodies to $\alpha$-toxin and NetB toxin; (5) levels of gene transcripts encoding pro-inflammatory cytokines and chemokines in the intestine and spleen. Infected chickens fed the XT-supplemented diet had increased body weight and reduced gut lesion scores compared with infected birds given the non-supplemented diet. The XT-fed group also displayed decreased serum $\alpha$-toxin levels and reduced intestinal $I L$ - 8 , lipopolysaccharide-induced TNF- $\alpha$ factor $(L I T A F), I L-17 A$ and $I L-17 F$ mRNA levels, while cytokine/chemokine levels in splenocytes increased in the XT-fed group, compared with the animals fed the control diet. In conclusion, the present study documents the molecular and cellular immune changes following dietary supplementation with extracts of Capsicum and turmeric that may be relevant to protective immunity against avian NE.
\end{abstract}

Key words: Capsicum: Turmeric: Chickens: Necrotic enteritis: Immunology

Necrotic enteritis (NE) and avian coccidiosis are among the most important infectious diseases in commercial poultry production, both resulting in field outbreaks with substantial mortality $^{(1-4)}$. The aetiological agent of $\mathrm{NE}$ is Clostridium perfringens, a Gram-positive, anaerobic, spore-forming bacterium that is transmitted by the faecal-oral route as well as through contaminated feed, water, housing structures and insects $^{(5-8)}$. The worldwide incidence of $C$. perfringensassociated NE has significantly increased in the last decade, primarily related to the decreasing use of in-feed antibiotics as growth promoters and antimicrobials. NE costs the international poultry industry approximately 2 billion US dollars annually because of medical treatments and impaired bodyweight gain ${ }^{(4,9,10)}$. NE is difficult to reproduce experimentally by infection with $C$. perfringens alone ${ }^{(11,12)}$ and several factors that promote the development of experimental disease have been identified, including a high-protein diet and co-infection with Eimeria, the aetiological agent of coccidiosis. Better understanding of pathogen-pathogen (Clostridium-Eimeria) as well as host-pathogen interactions is needed to develop rational, alternative and integrated management strategies to prevent both $\mathrm{NE}$ and coccidiosis ${ }^{(8,13)}$.

$\mathrm{NE}$ and coccidiosis have traditionally been prevented by antibiotics and coccidiostats ${ }^{(14-17)}$. However, recent interest has focused on developing drug-free disease control strategies due to the emergence of drug-resistant pathogens. Vaccination with attenuated strains of $C$. perfringens offers one approach to control avian $\mathrm{NE}^{(18)}$. Alternatively, potentiating immune effector mechanisms against NE using dietary phytonutrients may be feasible, although this approach has not been reported

Abbreviations: GAPDH, glyceraldehyde 3-phosphate dehydrogenase; LITAF, lipopolysaccharide-induced TNF- $\alpha$ factor; mAb, monoclonal antibody; NE, necrotic enteritis; NetB, necrotic enteritis B-like; PBS-T, PBS containing 0.05\% Tween; TNFSF15, TNF superfamily 15; XT, Capsicum oleoresin and turmeric oleoresin.

*Corresponding author: H. S. Lillehoj, fax +1 301504 5103, email hyun.lillehoj@ars.usda.gov 
in the literature. Previously, we showed that in vitro proliferation of splenocytes, a marker of cell-mediated immunity, was increased upon culture with an extract of turmeric compared with cells treated with vehicle alone ${ }^{(19)}$. Further, a dietary mixture of Capsicum oleoresin and turmeric oleoresin increased body weight and antibody responses to a recombinant Eimeria protein in Eimeria tenella-infected chickens compared with a non-supplemented diet ${ }^{(1)}$. Therefore, we hypothesised that this phytochemical mixture also would enhance protective immunity in chickens against NE following experimental Eimeria and Clostridium co-infection. To test this hypothesis, chickens were fed from hatch with a normal diet or with a diet supplemented with Capsicum and turmeric oleoresins, and immunity against NE was compared between the experimental and control groups.

\section{Materials and methods}

\section{Experimental animals and diets}

Broiler chickens (1 d old, Ross/Ross, Longenecker's Hatchery) were housed in Petersime starter brooder units and randomly assigned to three groups ( $n 15$ per group). Chickens were maintained in a temperature-controlled environment at $30^{\circ} \mathrm{C}$ for the first $2 \mathrm{~d}$ of growth followed by a gradual reduction in the temperature to $23^{\circ} \mathrm{C}$ until the end of the experiment, and continuously fed from hatch with a standard diet or the standard diet supplemented with XT (4 mg Capsicum oleoresin/ $\mathrm{kg}$ and $4 \mathrm{mg}$ turmeric oleoresin/ $\mathrm{kg}$ ) ad libitum for $20 \mathrm{~d}$. The concentration of the supplements in the diet was based on our previous report ${ }^{(1)}$. To facilitate the development of NE, birds were fed an antibiotic-free, certified organic starter diet containing $17 \%$ crude protein and $61 \%$ carbohydrate between days 1 and 18 after hatching and a standard grower diet containing $24 \%$ crude protein and 54\% carbohydrate between days 18 and 20. All the diets contained $15 \%$ vitamin and mineral mixture, $4.7 \%$ fat and $2 \cdot 4 \%$ fibre (US Department of Agriculture/Feed Mill). All the experiments were approved by the US Department of AgricultureAgricultural Research Service Institutional Animal Care and Use Committee.

\section{Necrotic enteritis disease model}

Chickens were kept in brooder pens in an Eimeria-free facility for $14 \mathrm{~d}$ post-hatch and transferred into large hanging cages ( $n 2$ birds per cage) at a separate location where they were infected and kept until the end of the experimental period. Chickens were orally infected on day 14 with Eimeria maxima strain $41 \mathrm{~A}\left(1.0 \times 10^{4}\right.$ oocysts/bird $)$ and on day 18 with $C$. perfringens strain Del- $\left(1.0 \times 10^{9}\right.$ colony-forming units/bird), a field isolate from a commercial poultry flock with endemic NE and a strain used in the NE disease model, which produces NE B-like (NetB) toxin ${ }^{(12,13,20)}$.

\section{Body weight and gut lesion scores}

Body weights were measured at day 14 (before infection with E. maxima) and day 20 post-hatch (day 6 post-infection with E. maxima and day 2 post-infection with C. perfringens) as described previously ${ }^{(2)}$. Cervical dislocation was used to euthanise the birds by well-trained personnel. At day 20 post-hatch, two equal sections of $10 \mathrm{~cm}$ located anterior and posterior to the diverticulum were collected for gut lesion scoring to evaluate the severity of NE symptoms on a scale of 0 (none) to 4 (high) in a blinded fashion by three independent observers as described earlier ${ }^{(21)}$.

\section{Preparation of serum for clostridial toxin and toxin antibody levels}

Blood samples ( $n 4$ birds per group) were collected by cardiac puncture immediately following euthanasia and sera were prepared by centrifugation at $1000 \mathrm{rpm}$ for $20 \mathrm{~min}$ at $4^{\circ} \mathrm{C}$. Sera in each group were used in ELISA to measure $\alpha$-toxin and NetB toxin levels, and toxin-specific antibody levels as described previously ${ }^{(22)}$.

\section{Cloning, expression and purification of recombinant Clostridium perfringens proteins}

Full-length coding sequences for genes encoding C.perfringens (ATCC 13124; American Type Culture Collection) $\alpha$-toxin and NetB toxin were cloned by PCR into the pET32a(+) vector with an $\mathrm{NH}_{2}$-terminal polyhistidine epitope tag as described previously ${ }^{(12,20)}$. Cloned genes were transformed into BL21(DE3) chemically competent E. coli (Invitrogen). The bacteria were cultured for $16 \mathrm{~h}$ at $37^{\circ} \mathrm{C}$, and induced for $5 \mathrm{~h}$ at $37^{\circ} \mathrm{C}$ with $1.0 \mathrm{~mm}$-isopropyl $\beta$-D-thiogalactopyranoside (Amresco). Thereafter, the bacteria were harvested by centrifugation at $10000 \mathrm{rpm}$ for $10 \mathrm{~min}$ at $4^{\circ} \mathrm{C}$, resuspended in PBS, disrupted by sonication and centrifuged at $10000 \mathrm{rpm}$ for $15 \mathrm{~min}$. The supernatant was incubated for $1 \mathrm{~h}$ at $22^{\circ} \mathrm{C}$ with Ni-NTA agarose (Qiagen), the resin was washed with PBS and purified clostridial proteins were eluted with $250 \mathrm{~mm}$-imidazole in PBS ( $\mathrm{pH}$ 9.2). Protein purity was confirmed by Coomassie blue-stained SDS-acrylamide gels.

\section{Monoclonal antibody production and validation of antigen specificity by ELISA}

Mouse monoclonal antibody (mAb) were produced using the previously described protocol ${ }^{(23-25)}$. BALB/c mice (National Cancer Institute) were immunised biweekly by intraperitoneal and subcutaneous injections with $50 \mu \mathrm{g}$ of recombinant proteins in $50 \%(\mathrm{v} / \mathrm{v})$ Freund's complete adjuvant (Sigma), and a final boost injection was given intravenously with $25 \mu \mathrm{g}$ of each protein without the adjuvant $3 \mathrm{~d}$ before fusion. Mice producing high serum antibody titres were selected by $\alpha$-toxin- or NetB toxin-specific ELISA, their splenic lymphocytes fused with SP2/0 cells (American Type Culture Collection) and hybridomas selected in medium supplemented with hypoxanthine, aminopterin and thymidine (all from Sigma) with a final confirmation of binding in ELISA as described previously $^{(23-25)}$. Briefly, ninety-six-well microtitre plates (Nunc) were coated overnight with $1.0 \mu \mathrm{g} /$ well of purified recombinant $\alpha$-toxin or NetB toxin proteins. The plates were washed 
with PBS containing $0.05 \%$ Tween (PBS-T) and blocked with PBS containing $1 \%$ bovine serum albumin. The mAb $(100 \mu \mathrm{l} /$ well) were incubated with agitation for $1 \mathrm{~h}$ at room temperature, washed with PBS-T and bound antibodies detected with peroxidase-conjugated rabbit anti-mouse IgG secondary antibody, 3,3',5,5'-tetramethylbenzidine and $\mathrm{H}_{2} \mathrm{O}_{2}$ (all from Sigma). Optical densities at $450 \mathrm{~nm}$ were determined with a microplate reader (Bio-Rad). Each $\mathrm{mAb}$ was purified using the Protein A IgG Purification Kit (Thermo Scientific) and diluted to $5.0 \mu \mathrm{g} / \mathrm{ml}$ in $50 \mathrm{~mm}$-carbonate buffer ( $\mathrm{pH}$ 9.6). mAb to $\alpha$-toxin or NetB toxin were used for the development of $\alpha$-toxin or NetB toxin capture ELISA.

\section{Production of polyclonal antisera to recombinant $\alpha$-toxin and necrotic enteritis $B$-like toxin proteins}

Rabbits were injected subcutaneously with $50 \mu \mathrm{g}$ of recombinant chicken $\alpha$-toxin or NetB toxin emulsified in $50 \%(\mathrm{v} / \mathrm{v})$ Freund's complete adjuvant. At 4 weeks later, rabbits were injected intramuscularly with $50 \mu \mathrm{g} \alpha$-toxin or NetB toxin emulsified in $50 \%(\mathrm{v} / \mathrm{v})$ Freund's incomplete adjuvant (Sigma). Rabbits were screened for anti- $\alpha$-toxin or NetB toxin antibodies by ELISA as described previously ${ }^{(26)}$. Once the anti- $\alpha$-toxin or NetB toxin titre had peaked, immune sera were collected and stored at $-20^{\circ} \mathrm{C}$. Rabbit anti- $\alpha$-toxin or -NetB toxin sera were conjugated with peroxidase using the EZ Link Plus Activated Peroxidase Kit (Thermo Scientific) following antibody purification using the Protein A IgG Purification Kit (Thermo Scientific).

\section{Capture ELISA for determining $\alpha$-toxin or necrotic enteritis $B$-like toxin concentrations}

$\mathrm{mAb}$ to $\alpha$-toxin or NetB toxin were coated at $0.5 \mu \mathrm{g} /$ well onto ninety-six well microtitre plates overnight at $4^{\circ} \mathrm{C}$. The plates were blocked and washed as described previously. Chicken sera were diluted 1:2 in PBS-T, $100 \mu \mathrm{l}$ added to the wells and incubated for $2 \mathrm{~h}$ at room temperature. The plates were washed with PBS-T, and $100 \mu \mathrm{l} /$ well of peroxidase-conjugated rabbit anti- $\alpha$-toxin or NetB toxin antibodies were added and incubated for $30 \mathrm{~min}$ followed by development with $3,3^{\prime}, 5,5^{\prime}$-tetramethylbenzidine substrate as described previously ${ }^{(26,27)}$. Optical densities at $450 \mathrm{~nm}$ were measured and serum $\alpha$-toxin or NetB toxin concentrations were determined using a standard curve generated with known concentrations of each purified recombinant protein.

\section{Serum antibody levels to $\alpha$-toxin and necrotic enteritis B-like toxin}

Sera were used in ELISA to measure $\alpha$-toxin- and NetB toxin-specific antibody levels as described previously ${ }^{(22)}$. Briefly, ninety-six-well microtitre plates were coated overnight with $1.0 \mu \mathrm{g} /$ well of purified recombinant $\alpha$-toxin or NetB toxin proteins. The plates were washed with PBS-T and blocked with PBS containing 1\% bovine serum albumin. Serum samples were diluted 1:20, $100 \mu \mathrm{l} /$ well incubated with agitation for $2 \mathrm{~h}$ at room temperature and washed with PBS-T. Bound antibodies were detected with peroxidase-conjugated rabbit anti-chicken IgG secondary antibody and 3,3',5,5'-tetramethylbenzidine substrate.

\section{Cytokine/chemokine transcript levels in the intestine and spleen}

At $2 \mathrm{~d}$ post-infection with $C$. perfringens, $20 \mathrm{~cm}$-long jejunum tissues located proximal to the Meckel's diverticulum and spleens were collected. The jejunum was cut open longitudinally, gently washed with ice-cold Hank's balanced salt solution (Sigma) and the mucosal layer was carefully scraped away using a surgical scalpel. Spleens collected on the same day were used for the preparation of single-cell suspensions. Whole spleens were washed with Hank's balanced salt solution, pressed gently through stainless-steel screens

Table 1. Oligonucleotide primers used for quantitative RT-PCR

\begin{tabular}{|c|c|c|c|}
\hline RNA target & Primer sequences & PCR product size (bp) & GenBank accession no. \\
\hline \multicolumn{4}{|l|}{ GAPDH } \\
\hline Forward & 5'-GGTGGTGCTAAGCGTGTTAT-3' & 264 & K01458 \\
\hline Reverse & 5'-АССТСТGTСАТСТСТССАСА-З' & & \\
\hline \multicolumn{4}{|l|}{ IL-8 } \\
\hline Forward & 5'-GGCTTGCTAGGGGAAATGA-3' & 200 & AJ009800 \\
\hline Reverse & 5'-AGCTGACTCTGACTAGGAAACTGT-3' & & \\
\hline \multicolumn{4}{|l|}{ LITAF } \\
\hline Forward & 5'-TGTGTATGTGCAGCAACCCGTAGT-3' & 229 & AY765397 \\
\hline Reverse & 5'-GGCATTGCAATTTGGACAGAAGT-3' & & \\
\hline \multicolumn{4}{|l|}{ TNFSF15 } \\
\hline Forward & 5'-CCTGAGTATTCCAGCAACGCA-3' & 292 & NM_01024578 \\
\hline Reverse & 5'-ATCCACCAGCTTGATGTCACTAAC-3' & & \\
\hline \multicolumn{4}{|c|}{ 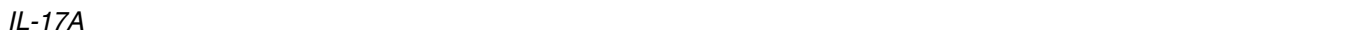 } \\
\hline Forward & 5'-CTCCGATCCCTTATTCTCСTC-3' & 292 & AJ493595 \\
\hline Reverse & 5'-AAGCGGTTGTGGTCCTCAT-3' & & \\
\hline \multicolumn{4}{|l|}{ IL-17F } \\
\hline Forward & 5'-TGAAGACTGCCTGAACCA-3' & 117 & JQ776598 \\
\hline Reverse & 5'-AGAGACCGATTCCTGATGT-3' & & \\
\hline
\end{tabular}

GAPDH, glyceraldehyde 3-phosphate dehydrogenase; LITAF, lipopolysaccharide-induced TNF- $\alpha$ factor; TNFSF15, TNF superfamily 15. 

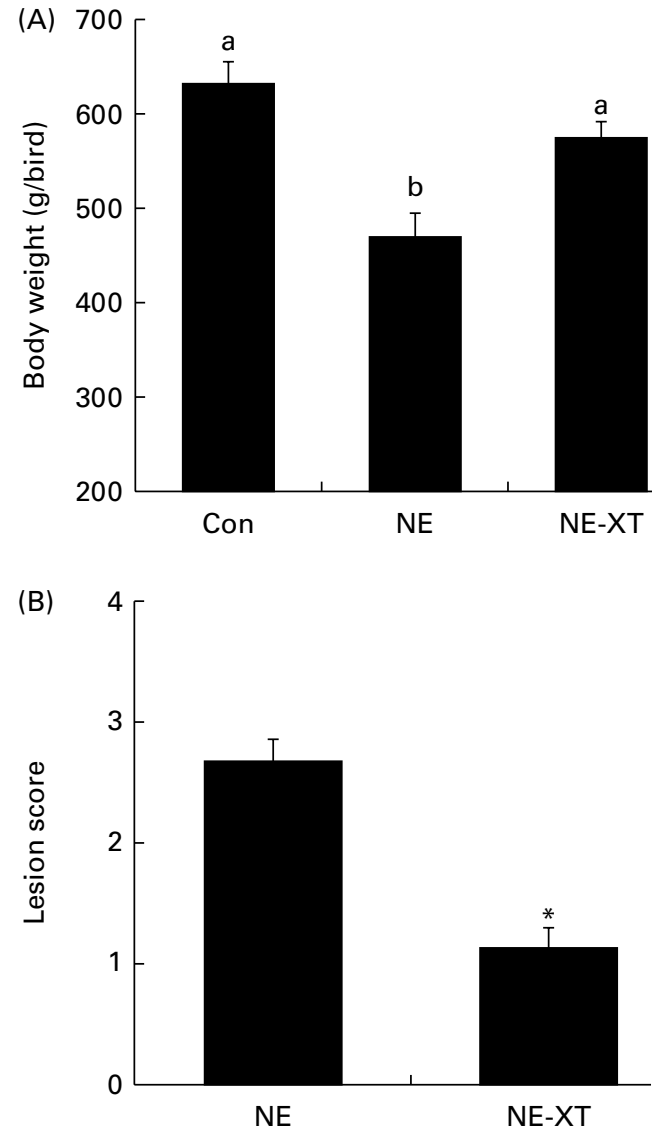

Fig. 1. Effect of dietary Capsicum oleoresin and turmeric oleoresin (XT) on (A) body weights and (B) intestinal lesion scores. Chickens were fed from hatch with a non-supplemented diet (Con) or a diet supplemented with $4 \mathrm{mg}$ Capsicum oleoresin $/ \mathrm{kg}$ and $4 \mathrm{mg}$ turmeric oleoresin/ $\mathrm{kg}$. At $14 \mathrm{~d}$ post-hatch, chickens were uninfected (Con) or orally infected with $1.0 \times 10^{4}$ sporulated oocysts of Eimeria maxima (necrotic enteritis (NE), NE-XT). At $18 \mathrm{~d}$ post-hatch, E. maxima-infected chickens were orally infected with $1.0 \times 10^{9}$ colony-forming units of Clostridium perfringens. (A) Body weights were measured at day 20. (B) Gut lesion scores were determined at day 20 Values are means, with their standard errors represented by vertical bars ( $n$ 15). ${ }^{\mathrm{a}, \mathrm{b}}$ Mean values with unlike letters were significantly different $\left(P<0.05\right.$; Duncan's multiple range test). ${ }^{*}$ Mean value was significantly different from that of the NE group $(P<0.05)$.
(Bio-World) into a Petri dish containing Hank's balanced salt solution and flushed through a cell strainer (BD Falcon) into a tube. Total RNA from the jejunum and spleen cells was extracted using TRIzol (Invitrogen). Then, $5 \mu \mathrm{g}$ of total RNA were treated with $1.0 \mathrm{U}$ of DNase I and $1.0 \mu \mathrm{l}$ of $10 \times$ reaction buffer (Sigma), incubated for $15 \mathrm{~min}$ at room temperature, $1.0 \mu \mathrm{l}$ of stop solution was added to inactivate DNase I and the mixture was heated for $10 \mathrm{~min}$ at $70^{\circ} \mathrm{C}$. RNA was reversetranscribed using the StrataScript first-strand synthesis system (Stratagene) according to the manufacturer's recommendations. Pro-inflammatory cytokine/chemokine transcript levels, which regulate host immunity against multiple pathogens, were analysed. Quantitative RT-PCR oligonucleotide primers for chicken $I L-8$, lipopolysaccharide-induced TNF- $\alpha$ factor (LITAF), TNF superfamily 15 (TNFSF15), IL-17A,IL-17F and glyceraldehyde 3-phosphate dehydrogenase (GAPDH) as an internal control are listed in Table 1. Amplification and detection were carried out using equivalent amounts of total RNA with the Mx3000P system and Brilliant SYBR Green qPCR master mix (Stratagene). Standard curves were generated using $\log _{10}$ diluted standard RNA and the levels of individual transcripts were normalised to those of GAPDH by the Q-gene program (http://www.gene-quantification.de/ download.html\#qgene $)^{(28)}$. Each sample was analysed in triplicate. To normalise individual replicates, logarithmic-scaled threshold cycle $\left(C_{\mathrm{t}}\right)$ values were transformed to linear units of normalised expression before calculating means and standard errors for references and individual targets, followed by the determination of mean normalised expression using the Q-gene program.

\section{Statistical analysis}

All data were subjected to one-way ANOVA using SPSS 15.0 for Windows (SPSS, Inc.). Mean values of the treatment groups were compared using Duncan's multiple range test or the $t$ test, and differences were considered as statistically significant at $P<0 \cdot 05$.

Table 2. Effect of dietary Capsicum oleoresin and turmeric oleoresin (XT) on the levels of serum $\alpha$-toxin, necrotic enteritis (NE) B-like (NetB) toxin and antibodies to $\alpha$-toxin and NetB toxin (Mean values with their standard errors, $n 4$ )

\begin{tabular}{|c|c|c|c|c|c|c|c|c|}
\hline \multirow[b]{3}{*}{ Groups } & \multicolumn{4}{|c|}{ Toxint } & \multicolumn{4}{|c|}{ Ab titre $\ddagger$} \\
\hline & \multicolumn{2}{|c|}{$\alpha$-Toxin (ng/ml) } & \multicolumn{2}{|c|}{ NetB (ng/ml) } & \multicolumn{2}{|c|}{$\alpha$-Toxin (OD) } & \multicolumn{2}{|c|}{ NetB (OD) } \\
\hline & Mean & SEM & Mean & SEM & Mean & SEM & Mean & SEM \\
\hline $\mathrm{NE}$ & $780 \cdot 8$ & 112.4 & 931.6 & 72.9 & 0.95 & 0.14 & 0.87 & 0.04 \\
\hline NE-XT & $382 \cdot 9^{*}$ & $64 \cdot 3$ & $886 \cdot 3$ & $100 \cdot 5$ & $1 \cdot 22$ & 0.10 & 1.01 & 0.09 \\
\hline \multicolumn{9}{|c|}{$\begin{array}{l}\text { Ab, antibody; OD, optical density. } \\
{ }^{*} \text { Mean value was significantly different from that of the NE group }(P<0.05) \text {. } \\
\text { † Chickens were fed with the non-supplemented or XT-supplemented diet and co-infected with Clostridium } \\
\text { perfringens and Eimeria maxima. Sera were collected at day } 20 \text { and used to measure the levels of } \alpha \text {-toxin } \\
\text { and NetB toxin by ELISA. }\end{array}$} \\
\hline
\end{tabular}




\section{Results}

Effects of dietary supplementation with Capsicum oleoresin and turmeric oleoresin on body weights and intestinal lesion scores

Initially, we determined that chickens fed the XT phytonutrient mixture did not exhibit gross pathological effects when used at the denoted concentrations. Further, continuous feeding with the XT-supplemented diet during the first $14 \mathrm{~d}$ post-hatch and before E. maxima infection did not affect body weight compared with the non-supplemented diet group (data not shown). Chickens fed the XT-supplemented diet and co-infected with E. maxima and C.perfringens exhibited increased body weight at $20 \mathrm{~d}$ post-hatch compared with the infected animals given the non-supplemented diet (Fig. 1(A)). Indeed, there was no significant difference in weight between the non-supplemented, non-infected controls and the infected chickens fed the XT-supplemented diet. Birds fed the XT diet and co-infected with E. maxima and C.perfringens also showed significantly reduced intestinal lesion scores at $2 \mathrm{~d}$ post-infection with $C$. perfringens compared with the non-supplemented and infected controls (Fig. 1(B)).

\section{Effect of dietary supplementation with Capsicum oleoresin and turmeric oleoresin on serum toxin and toxin antibody levels}

The $\alpha$-toxin and NetB toxin were not detected in the serum of the control groups (data not shown). Serum $\alpha$-toxin levels were significantly lower in the infected XT group compared with the non-supplemented and infected controls (Table 2). However, no significant difference was found in serum NetB toxin levels between the two groups (Table 2). While there was a trend towards higher serum antibody titres against the $\alpha$-toxin and NetB toxin in the XT group compared with the non-supplemented and infected groups, these differences were not statistically significant (Table 2).

Effect of dietary supplementation with Capsicum oleoresin and turmeric oleoresin on cytokine/chemokine transcript levels

IL-8, LITAF, TNFSF15, IL-17A and IL-17F transcript levels in the intestinal jejunum were increased in E. maxima/C. perfringens co-infected chickens on the non-supplemented diet compared with the non-supplemented, uninfected controls (Fig. 2). With the exception of TNFSF15 transcripts, all intestinal transcript levels were significantly reduced in infected chickens fed the XT-supplemented diet compared with the non-supplemented, infected controls. By contrast, in the spleen, only $I L-17 \mathrm{~A}$ transcripts were increased in E. maxima/C. perfringens co-infected chickens on the non-supplemented diet compared with the non-supplemented, uninfected controls, and the infected chickens fed the XT diet had increased levels of all transcripts compared with the non-supplemented, infected controls (Fig. 3).
(A)

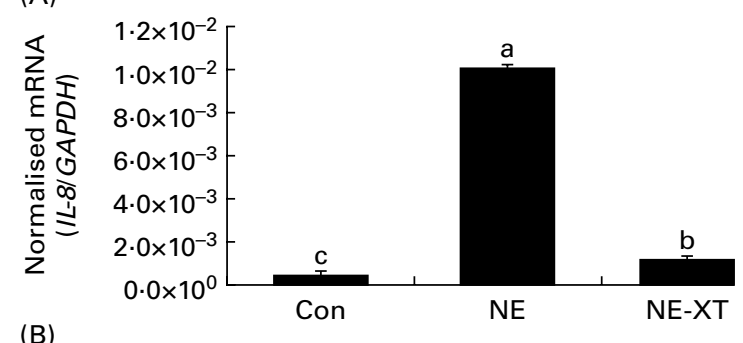

(B)

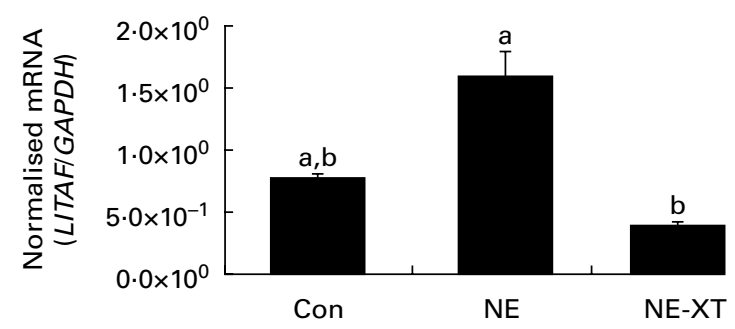

(C)

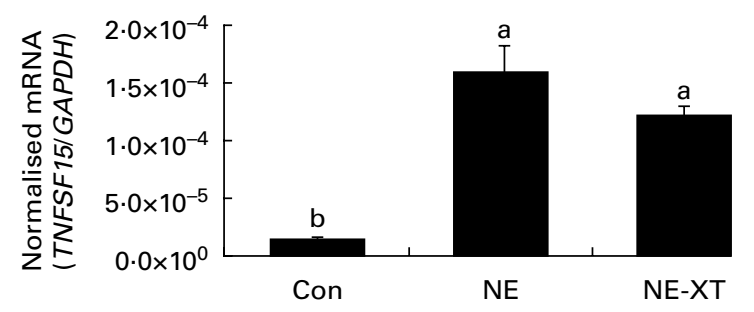

(D)

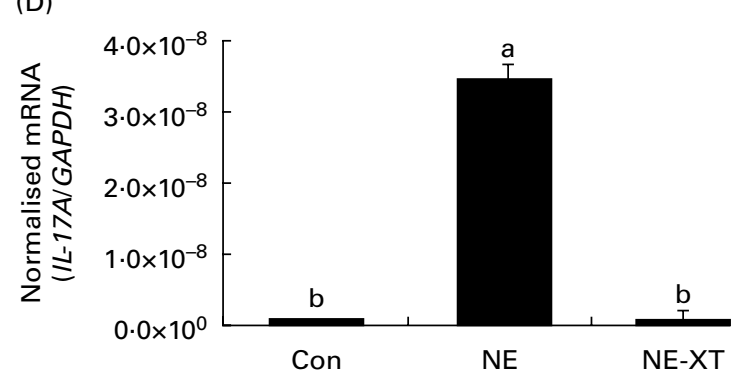

(1)

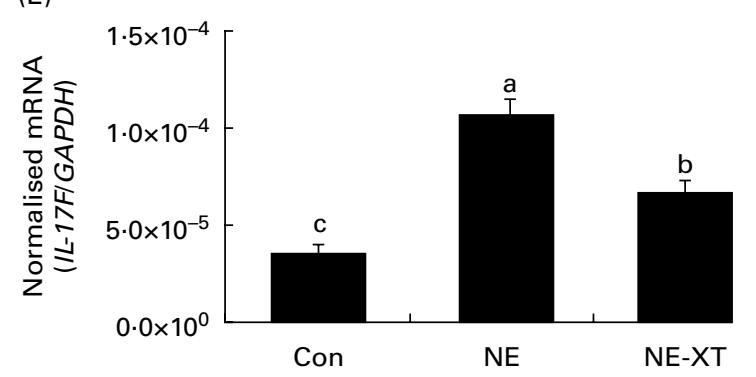

Fig. 2. Effect of dietary Capsicum oleoresin and turmeric oleoresin (XT) on the levels of cytokine/chemokine transcripts in the intestine. Chickens were fed with the non-supplemented (Con) or XT-supplemented diet and uninfected or co-infected with Clostridium perfringens and Eimeria maxima as described in Fig. 1. Jejunum intestinal epithelia were collected at day 20 and used to measure the levels of transcripts for $(A) I L-8$, (B) lipopolysaccharideinduced TNF- $\alpha$ factor (LITAF), (C) TNF superfamily 15 (TNFSF15), (D) $I L-17 A$ and $(E) I L-17 F$ by quantitative RT-PCR. Individual transcript levels were normalised to glyceraldehyde 3-phosphate dehydrogenase (GAPDH) transcripts. Values are means $(n 4)$, with their standard errors represented by vertical bars. ${ }^{a, b, c}$ Mean values with unlike letters were significantly different $(P<0.05$; Duncan's multiple range test). NE, necrotic enteritis. 
(A)

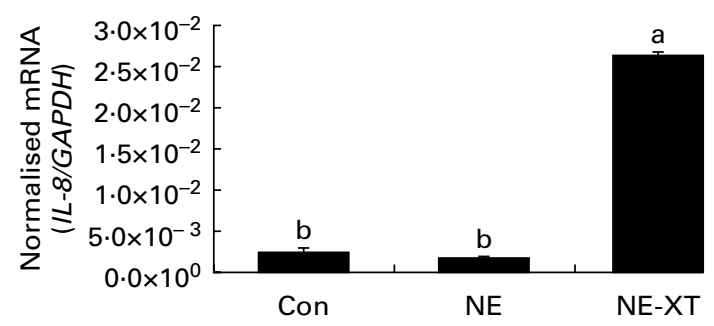

(B)

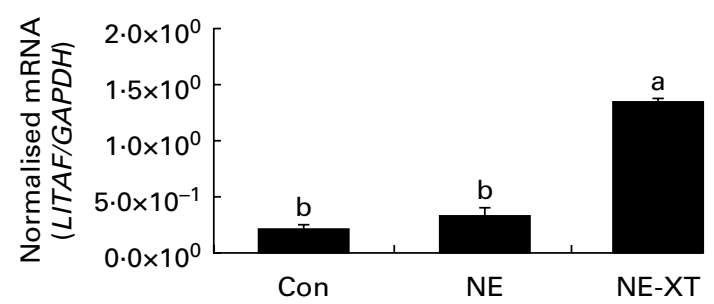

(C)

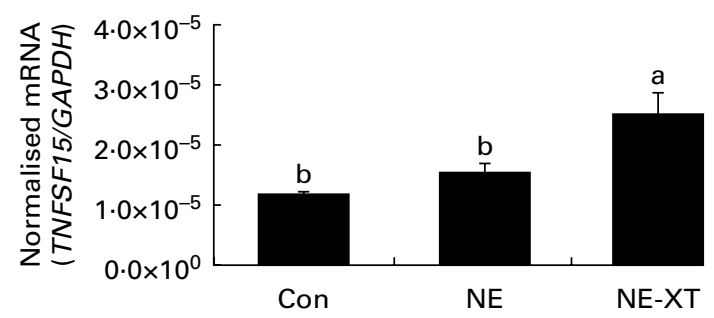

(D)

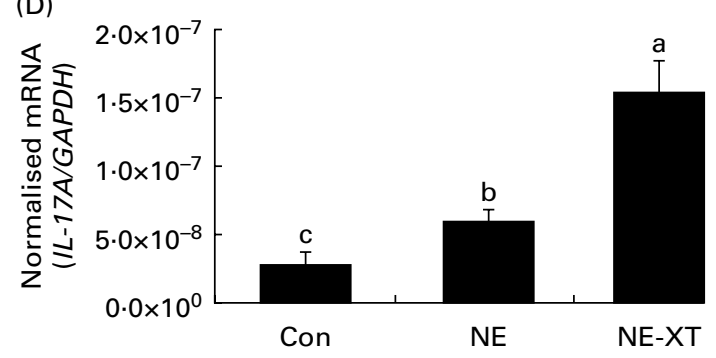

(E)

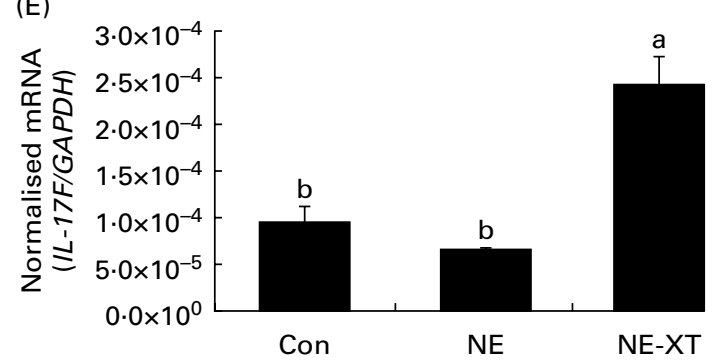

Fig. 3. Effect of dietary Capsicum oleoresin and turmeric oleoresin (XT) on the levels of cytokine/chemokine transcripts in the spleen. Chickens were fed with the non-supplemented (Con) or XT-supplemented diet and uninfected or co-infected with Clostridium perfringens and Eimeria maxima as described in Fig. 1. Spleen cells were collected at day 20 and used to measure the levels of transcripts for (A) $I L-8$, (B) lipopolysaccharide-induced TNF- $\alpha$ factor (LITAF), (C) TNF superfamily 15 (TNFSF15), (D) IL-17A and (E) IL-17F by quantitative RT-PCR. Individual transcript levels were normalised to glyceraldehyde 3-phosphate dehydrogenase (GAPDH) transcripts. Values are means $(n 4)$, with their standard errors represented by vertical bars. $\mathrm{a}, \mathrm{b}, \mathrm{c}$ Mean values with unlike letters were significantly different $(P<0.05$; Duncan's multiple range test). NE, necrotic enteritis.

\section{Discussion}

The present study was conducted to evaluate the effect of dietary extracts of Capsicum and turmeric oleoresins on local and systemic immune responses against experimental NE using a Clostridium/Eimeria co-infection model system in commercial broilers. To induce NE symptoms approximating those seen in field infections, the $C$. perfringens strain, co-infection with Eimeria, and dietary factors were utilised $^{(8,12,18)}$. Chickens fed the XT-supplemented diet and co-infected with $C$. perfringens and E. maxima showed significantly (1) increased body weight, (2) reduced gut lesions, (3) decreased serum $\alpha$-toxin levels and (4) reduced $I L-8$, LITAF, IL-17A and $I L-17 F$ mRNA levels in the intestine, but increased cytokine/chemokine mRNA levels in splenocytes, compared with infected chickens given the non-supplemented control diet. These collective results suggest that XT has a protective effect against experimental NE. The protective properties of dietary XT against NE were originally hypothesised on the basis of our previous reports of direct cytotoxic effects against Eimeria and the heightened immune responses against avian coccidiosis by certain phytonutrients, including Capsicum and turmeric oleoresins ${ }^{(1,29)}$.

The XT group showed slightly higher body weights compared with the control group at $14 \mathrm{~d}$ and before infection with E. maxima and C. perfringens (data not shown). Following infection, however, the XT group exhibited significantly increased weight compared with the unsupplemented control group $(P<0.05)$. Increased body weight and/or decreased intestinal lesions in the XT-fed group may be attributable to improved intestinal physiology, allowing for greater nutrient absorption compared with chickens given the nonsupplemented control diet. A similar effect was proposed previously to account for the protective effect of XT on vaccine-stimulated immunity in broiler chickens against infection by Eimeria tenella ${ }^{(1)}$.

C. perfringens $\alpha$-toxin, while initially reported as a major virulence factor in chickens ${ }^{(5,30)}$, is now considered as non-essential for producing disease ${ }^{(5)}$. Nevertheless, chicken antibodies against $\alpha$-toxin may be considered as markers of infection $^{(31)}$, which is consistent with the observed decrease in serum $\alpha$-toxin levels in XT-fed birds compared with the non-supplemented controls. Given that $\alpha$-toxin antibody levels were equal in the two groups, it is unlikely that the diminished $\alpha$-toxin levels can be attributed to host antibodies masking the capture and/or detection antibodies used in the ELISA protocol. This possibility, however, may be relevant to the situation with NetB toxin levels where no differences between the experimental and control groups were noted. Another consideration may relate to differential kinetics of the two antibody responses, and examination of toxin antibody levels at greater times post-infection may reveal altered NetB toxin levels in the XT-supplemented group $v$. controls.

The intestinal microflora plays a critical role in inflammatory gut diseases of humans and animals. More specifically, an imbalance in the mucosal cytokine profile causes transient intestinal inflammation following initial exposure to faecal bacteria or their antigens ${ }^{(32)}$. The present results suggest that 
XT regulates cellular immunity by altering the expression levels of these pro-inflammatory cytokine and chemokine genes in the intestine of infected animals. This is consistent with previous studies which demonstrated that dietary supplementation of chickens with Capsicum oleoresin reduced coccidiosis symptoms and altered the in vivo expression of a family of innate immune response genes compared with chickens fed a non-supplemented diet ${ }^{(1,29,33)}$. Some of the transcripts encoded by these genes were included for analysis in the present report. IL- 8 is a CXC chemokine that attracts leucocytes, primarily neutrophils, to mucosal sites of inflammation. LITAF is a pro-inflammatory cytokine expressed by lymphoid cells, including peripheral blood, lymph node and intestinal leucocytes, that regulates host immunity against multiple pathogens ${ }^{(34)}$. TNFSF15 is also abundantly expressed by immune cells and is significantly involved in their differentiation, proliferation and apoptosis ${ }^{(35)}$. IL-17A is a pro-inflammatory cytokine produced by activated $\mathrm{T}$ cells, which controls the activation of NF- $\mathrm{B}$ and mitogen-activated protein kinases, stimulates the expression of IL- 6 and cyclo-oxygenase- 2 and enhances the production of NO. High levels of IL-17A are associated with several chronic inflammatory diseases ${ }^{(36-38)}$. Another member of the IL-17 family, IL-17F, is expressed by activated $\mathrm{T}$ cells that acts by stimulating the production of IL-6, IL-8 and granulocyte macrophage colony-stimulating factor $(\mathrm{GM}-\mathrm{CSF})^{(39,40)}$.

Pro-inflammatory cytokines/chemokines regulate host immunity against multiple pathogens through immune cell differentiation, proliferation, apoptosis and NO production. Their continuous high levels may lead to intestinal damage and increased energy consumption. While all of the aforementioned mediators appear to act in the context of heightened inflammation, we observed that XT decreased their corresponding mRNA levels in the gut epithelium. Accordingly, their decreased intestinal levels may be related to the protective effect of XT treatment on improving intestinal health in the context of NE. Differential kinetics of expression of the individual cytokine/chemokine, their sites of production within the intestinal tract and their relative activities during avian NE may be relevant here. Interestingly, however, their expression levels significantly increased in the spleen. This result showed that expression of innate immune response genes works differently in different organs or systematically to maintain homeostasis in chickens exposed to NE disease.

In summary, a mixture of Capsicum and turmeric oleoresins was identified as an effective phytonutrient against clinical signs of experimental avian NE when supplied in dietary form. Future studies are needed to further define the molecular and cellular characteristics of this phytochemical combination for the control of NE in the field.

\section{Acknowledgements}

The authors thank Dr Kyung Woo Lee, Dr Duk Kyung Kim, Myeong Seon Park, Marjorie Nichols, Stacy O'Donnell and Ashley Cox for scientific input and technical assistance. The present study was partially supported by a formal trust agreement established between the Agricultural Research Service-
US Department of Agriculture and Pancosma S.A., and the World Class University Program (R33-10013) of the Ministry of Education, Science and Technology of South Korea. The present study was carried out during sabbatical leave at the National Academy of Agricultural Science, Rural Development Administration, Suwon, Gyeonggi, Korea. Mention of trade names or commercial products in this publication is solely for the purpose of providing specific information and does not imply recommendation or endorsement by the US Department of Agriculture. S. H. L. conceived, designed and performed the experiments, analysed the data, and wrote the manuscript; H. S. L. conceived and designed the experiments; S. I. J. performed the experiments; M. S. J. performed the experiments; E. P. L. wrote the manuscript; W. M. designed the experiments; D. M. B. conceived the experiments. The authors declare that there are no conflicts of interest.

\section{References}

1. Lee SH, Lillehoj HS, Jang SI, et al. (2011) Effects of dietary supplementation with phytonutrients on vaccine-stimulated immunity against infection with Eimeria tenella. Vet Parasitol 181, 97-105.

2. Lee SH, Lillehoj HS, Jang SI, et al. (2011) Cinnamaldehyde enhances in vitro parameters of immunity and reduces in vivo infection against avian coccidiosis. Br J Nutr 106, 862-869.

3. Lovland A \& Kaldhusdal M (2001) Severely impaired production performance in broiler flocks with high incidence of Clostridium perfringens-associated hepatitis. Avian Pathol 30, 73-81.

4. Van Immerseel F, Rood JI, Moore RJ, et al. (2009) Rethinking our understanding of the pathogenesis of necrotic enteritis in chickens. Trends Microbiol 17, 32-36.

5. Keyburn AL, Sheedy SA, Ford ME, et al. (2006) Alpha-toxin of Clostridium perfringens is not an essential virulence factor in necrotic enteritis in chickens. Infect Immun $\mathbf{7 4}$, 6496-6500.

6. Songer JG \& Uzal FA (2005) Clostridial enteric infections in pigs. J Vet Diagn Invest 17, 528-536.

7. Van Immerseel F, De Buck J, Pasmans F, et al. (2004) Clostridium perfringens in poultry: an emerging threat for animal and public health. Avian Pathol 33, 537-549.

8. Williams RB (2005) Intercurrent coccidiosis and necrotic enteritis of chickens: rational, integrated disease management by maintenance of gut integrity. Avian Pathol 34, 159-180.

9. Cooper KK, Trinh HT \& Songer JG (2009) Immunization with recombinant $\alpha$ toxin partially protects broiler chicks against experimental challenge with Clostridium perfringens. Vet Microbiol 133, 92-97.

10. Van der Sluis W (2000) Clostridial enteritis is an often underestimated problem. World Poult 16, 42-43.

11. Collier CT, Hofacre CL, Payne AM, et al. (2008) Coccidiainduced mucogenesis promotes the onset of necrotic enteritis by supporting Clostridium perfringens growth. Vet Immunol Immunopathol 122, 104-115.

12. Park SS, Lillehoj HS, Allen PC, et al. (2008) Immunopathology and cytokine responses in broiler chickens coinfected with Eimeria maxima and Clostridium perfringens with the use of an animal model of necrotic enteritis. Avian Dis 52, 14-22.

13. Lee KW, Lillehoj HS, Jeong W, et al. (2011) Avian necrotic enteritis: experimental models, host immunity, pathogenesis, 
risk factors, and vaccine development. Poult Sci 90, 1381-1390.

14. Lillehoj HS \& Lillehoj EP (2000) Avian coccidiosis. A review of acquired intestinal immunity and vaccination strategies. Avian Dis 44, 408-425.

15. Lillehoj HS \& Lee SH (2007) Probiotics as an alternative control strategy against avian coccidiosis. Feedinfo News Service Scientific Reviews. http://www.feedinfo.com (accessed September 2007).

16. Lillehoj HS \& Lee SH (2007) Dietary modulation of intestinal innate immunity using plant-derived phytochemicals. Feedinfo News Service Scientific Reviews. http://www. feedinfo.com (accessed September 2007).

17. Lee SH, Lillehoj HS, Cho SM, et al. (2008) Immunomodulatory properties of dietary plum on coccidiosis. Comp Immunol Microb 31, 389-402.

18. Thompson DR, Parreira VR, Kulkarni RR, et al. (2006) Live attenuated vaccine-based control of necrotic enteritis of broiler chickens. Vet Microbiol 113, 25-34.

19. Lee SH, Lillehoj HS, Hong YH, et al. (2010) In vitro effects of plant and mushroom extracts on immunological function of chicken lymphocytes and macrophages. Br Poult Sci 51, 213-221.

20. Lee KW, Lillehoj HS, Li G, et al. (2011) Identification and cloning of two immunogenic Clostridium perfringens proteins, elongation factor $\mathrm{Tu}$ (EF-Tu) and pyruvate: ferredoxin oxidoreductase (PFO) of $C$. perfringens. Res Vet Sci 91, e80-e86.

21. Prescott JF (1979) The prevention of experimentally induced necrotic enteritis in chickens by avoparcin. Avian Dis 23, 1072-1074

22. Li G, Lillehoj HS, Lee KW, et al. (2010) An outbreak of gangrenous dermatitis in commercial broiler chickens. Avian Pathol 39, 247-253.

23. Lee SH, Lillehoj HS, Jang SI, et al. (2011) Development and characterization of mouse monoclonal antibodies reactive with chicken interleukin-2 receptor alpha chain (CD25). Vet Immunol Immunopathol 144, 396-404.

24. Lee SH, Lillehoj HS, Park MS, et al. (2011) Development and characterization of mouse monoclonal antibodies reactive with chicken CD80. Comp Immunol Microbiol 34, 273-279.

25. Lee SH, Lillehoj HS, Jang SI, et al. (2012) Development and characterization of mouse monoclonal antibodies reactive with chicken CD83. Vet Immunol Immunopathol $\mathbf{1 4 5}$ $527-533$.

26. Miyamoto T, Lillehoj HS, Sohn EJ, et al. (2001) Production and characterization of monoclonal antibodies detecting chicken interleukin-2 and the development of an antigen capture enzyme-linked immunosorbent assay. Vet Immunol Immunopathol 80, 245-257.

27. Huang Z, Fang J, Gu J, et al. (2011) Development of a capture ELISA to determine kinetics of soluble CD25 following in vitro and in vivo stimulation of duck peripheral blood monocytes. Vet Immunol Immunopathol 140, 102-109.

28. Muller PY, Janovjak H, Miserez AR, et al. (2002) Processing of gene expression data generated by quantitative real-time RT-PCR. Biotechniques 32, 1372-1379.

29. Lee SH, Lillehoj HS, Jang SI, et al. (2010) Effect of dietary Curcuma, Capsicum, and Lentinus on enhancing local immunity against Eimeria acervulina infection. J Poult Sci 47, 89-95.

30. Songer JG (1996) Clostridial enteric diseases of domestic animals. Clin Microbiol Rev 9, 216-234.

31. Keyburn AL, Bannam TL, Moore RJ, et al. (2010) NetB, a pore-forming toxin from necrotic enteritis strains of Clostridium perfringens. Toxin (Basal) 2, 1913-1927.

32. Sydora BC, MacFarlane SM, Lupicki M, et al. (2010) An imbalance in mucosal cytokine profile causes transient intestinal inflammation following an animal's first exposure to fecal bacteria and antigens. Clin Exp Immunol 161, 187-196.

33. Kim DK, Lillehoj HS, Lee SH, et al. (2010) High-throughput gene expression analysis of intestinal intraepithelial lymphocytes after oral feeding of carvacrol, cinnamaldehyde, or Capsicum oleoresin. Poult Sci 89, 68-81.

34. Hong YH, Lillehoj HS, Lee SH, et al. (2006) Molecular cloning and characterization of chicken lipopolysaccharideinduced TNF- $\alpha$ factor (LITAF). Dev Comp Immunol 30, 919-929.

35. Collette Y, Gilles A, Pontarotti P, et al. (2003) A co-evolution perspective of the TNFSF and TNFRSF families in the immune system. Trends Immunol 24, 387-394.

36. Kishimoto $\mathrm{T}$ (2005) Interleukin-6 from basic science to medicine - 40 years in immunology. Annu Rev Immunol 23, $1-21$.

37. Korn T, Bettelli E, Oukka M, et al. (2009) IL-17 and Th17 cells. Annu Rev Immunol 27, 485-517.

38. Kolls JK \& Linden A (2004) Interleukin-17 family members and inflammation. Immunity 21, 467-476.

39. Weaver CT, Hatton RD \& Mangan PR (2007) IL-17 family cytokines and the expanding diversity of effector $\mathrm{T}$ cell lineages. Annu Rev Immunol 25, 821-852.

40. Hirata T, Osuga Y, Takamura M, et al. (2011) Interleukin-17F increases the secretion of interleukin- 8 and the expression of cyclooxygenase 2 in endometriosis. Fertil Steril 96, 113-117. 